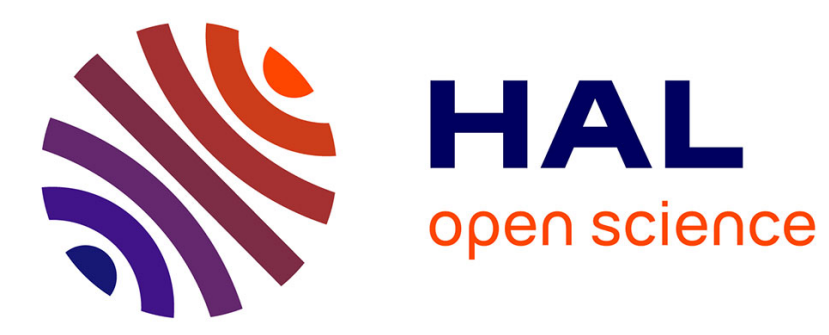

\title{
Independent component analysis within polarimetric incoherent target decomposition
}

Nikola Besic, Gabriel Vasile, Jocelyn Chanussot, Srdjan Stankovic, Didier Boldo, Guy d'Urso

\section{> To cite this version:}

Nikola Besic, Gabriel Vasile, Jocelyn Chanussot, Srdjan Stankovic, Didier Boldo, et al.. Independent component analysis within polarimetric incoherent target decomposition. IGARSS 2013 - IEEE International Geoscience and Remote Sensing Symposium, Jul 2013, Melbourne, Australia. pp.4158-4161. hal-00852109

\section{HAL Id: hal-00852109 \\ https://hal.science/hal-00852109}

Submitted on 20 Aug 2013

HAL is a multi-disciplinary open access archive for the deposit and dissemination of scientific research documents, whether they are published or not. The documents may come from teaching and research institutions in France or abroad, or from public or private research centers.
L'archive ouverte pluridisciplinaire HAL, est destinée au dépôt et à la diffusion de documents scientifiques de niveau recherche, publiés ou non, émanant des établissements d'enseignement et de recherche français ou étrangers, des laboratoires publics ou privés. 


\title{
INDEPENDENT COMPONENT ANALYSIS WITHIN POLARIMETRIC INCOHERENT TARGET DECOMPOSITION
}

\author{
N. Besic ${ }^{1,2}$, G. Vasile $^{1}$, J. Chanussot ${ }^{1,3}$, S. Stankovic ${ }^{2}$, D. Boldo ${ }^{4}$ and G. d'Urso ${ }^{4}$ \\ ${ }^{1}$ GIPSA-Lab, CNRS/Grenoble Institute of Technology, Grenoble, France \\ ${ }^{2}$ Faculty of Electrical Engineering, University of Montenegro, Podgorica, Montenegro \\ ${ }^{3}$ Faculty of Electrical and Computer Engineering, University of Iceland, Reykjavik, Iceland \\ ${ }^{4}$ R\&D STEP, Electricity of France (EDF), Chatou, France
}

\begin{abstract}
This paper represents a part of our efforts to generalize polarimetric incoherent target decomposition to the level of BSS techniques by introducing the ICA method instead of the conventional eigenvector decomposition. We compare, in the frame of polarimetric incoherent target decomposition, several criteria for the estimation of complex independent components $[1,2]$. This is done by parametrising the obtained dominant and mutually independent target vectors using the TSVM [3] and representing them on the corresponding Poincaré sphere. We demonstrate notably good performances of the proposed method applied on the RAMSES POLSAR X-band image, by precisely identifying the class of trihedral reflectors present in the scene. Logarithm and square root nonlinearities - two of the three proposed criteria for complex IC derivation prove to be very efficient. The best discrimination between the a priori defined classes appears to be achieved with the principal kurtosis criterion. Finally, the algorithm using the former two functions leads to very interesting entropy estimation.
\end{abstract}

Index Terms - Polarimetric ICTD, Complex Fast-ICA, TVSM, Poincaré sphere, non-gaussianity

\section{INTRODUCTION}

Conventional algebraic incoherent target decompositions are based on the Hermitian nature of the coherence (or covariance) matrix [4]. Eigenvector decomposition of the space averaged coherence matrix results in a set of mutually orthogonal target vectors, representing the dominant scatterers in a scene. Accompanying eigenvalues define the corresponding scatterers contribution to the total scattering. The two mostly used algebraic decompositions are the $H / \alpha$ decomposition, proposed by Cloude and Pottier [5] and the Touzi decomposition [3]. They differ in terms of target vector parametrization: the first one uses $\alpha / \beta$ model, while the second uses

Contact e-mail: nikola.besic@gipsa-lab.grenoble-inp.fr

This work was supported by the French National R\&DProgram TSEN-X PEPS UJF-CNRS 2012. the Target Scattering Vector Model (TSVM), which ensures roll-invariance in case of both symmetric and non-symmetric targets.

The Independent Component Analysis (ICA) is a blind source separation technique (BSS) aiming to recover independent source signals without having any physical knowledge of the mixing process [6]. Unlike the Principal Component Analysis (PCA), which is limited to the second order statistics of the observations and therefore results in statistically uncorrelated sources, ICA is rather based on the higher order statistics.

It can be shown that the eigenvector decomposition of the scattering coherence matrix, under certain constraints, provides the same results as the Principal Component Analysis (PCA) of the corresponding representative target vector [7]. The idea is to propose a generalization of the polarimetric decomposition to the level of blind source separation techniques by introducing several different ICA methods instead of the eigenvector decomposition. The principal motivation is the possibility to exploit higher order statistics of the nonGaussian target vector in order to recover a set of independent dominant scatterers. The recovered linearly independent scattering target vectors are not necessarily orthogonal. They are parametrised using the TSVM, allowing the Poincare sphere representation with direct physical interpretation [8].

In this paper we compare, in the framework of ICTD, different criteria used in the derivation of complex independent components, by applying Complex NC-FastICA algorithm [2]. The analysis is done by investigating the efficiency in: the identification of elementary reflectors in the scene, the discrimination between different targets and the entropy estimation.

\section{METHOD}

The first step is the statistical classification of the POLSAR image [9]. After initialization by $H / \alpha$ unsupervised classification, we compute the barycenters of the classes using the Riemannian distance in covariance space. Finally, pixels are 


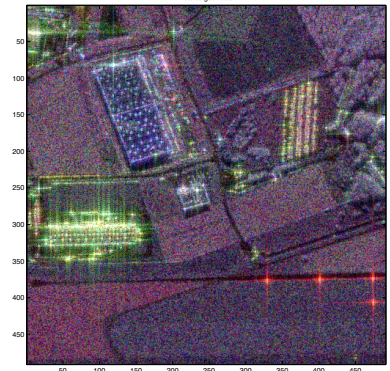

(a)

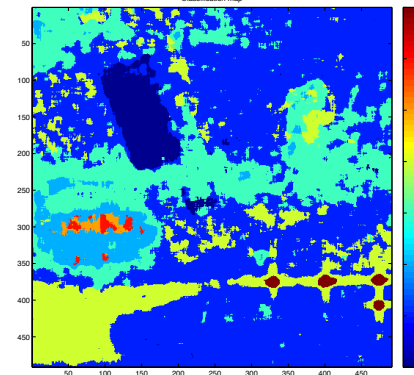

(b)
Fig. 1. RAMSES POLSAR X-band, Brétigny, France: (a) Pauli RGB coded image; (b) Statistical classification performed in the first step.

assigned using the Wishart criterion.

In this phase we obtain the set of representative target vectors $\mathbf{K}_{\mathbf{c}}$ for each of the classes $c$ (Fig. 1). Those vector sets represent the observation data for the BSS (eq. 1), while the selection method assures method relevance in the case of incoherent targets.

The core of the ICA based decomposition is the estimation of the mixing matrix $\mathbf{A}$ (Eq. 1). There are several criteria for determining the elements of $\mathbf{A}$ in order to ensure the mutual independence of the sources in $\mathbf{s}$. The common factor for all the applied methods is the assumption which proves to be reasonable in case of high resolution polarimetric SAR images [10] - at most one of the sources is Gaussian [6].

$$
\mathbf{k}^{c}(i, j)=\left[\begin{array}{lll}
A_{11}^{c} & A_{12}^{c} & A_{13}^{c} \\
A_{21}^{c} & A_{22}^{c} & A_{23}^{c} \\
A_{31}^{c} & A_{32}^{c} & A_{33}^{c}
\end{array}\right] \cdot\left[\begin{array}{l}
s_{1}^{c}(i, j) \\
s_{2}^{c}(i, j) \\
s_{3}^{c}(i, j)
\end{array}\right]=\mathbf{A}^{c} \mathbf{s}^{c}(i, j)
$$

We compare, in the framework of ICTD, the performances of several different strategies used in the estimation of the complex independent components. This is done by applying several different Complex NC-FastICA algorithm criteria [2].

The FastICA algorithm is a fast converging algorithm based on a fixed-point iteration scheme for finding a maximum of the nongaussianity of each of the sources $s=\mathrm{w}^{\mathbf{H}} \mathbf{X}$ [11], with $\mathbf{x}$ being the (whitened) observation data vector and $\mathbf{w}$ the mixing vector converging to one of the columns of the mixing matrix $\mathbf{A}$. In our case, the observation data are the Pauli target vectors corresponding to the $a$ priori defined class $\left(\mathbf{k}_{\mathbf{c}} \in \mathbf{K}_{\mathbf{c}}\right.$ ), meaning that we finally obtain one mixing matrix $\mathbf{A}^{c}$ for each of the classes $c$.

The Complex Fast-ICA algorithm is based on a bottomup approach: emphasizing the non-gaussanity of the sources by maximizing an arbitrary nonlinear contrast function (Eq. 2) whose extrema coincides with the independent component [1].

$$
J_{G}(\mathbf{w})=\mathbf{E}\left\{\mathbf{G}\left(\left|\mathbf{w}^{\mathbf{H}} \mathbf{x}\right|^{2}\right)\right\}
$$

The performances of the algorithm depend strongly on the choice of the nonlinear function $G(y)$, which is supposed to be suited to the particular application. Therefore, here we use three different functions, leading to different criteria $(C)$ in deriving independent target vectors:

- kurtosis $(\mathrm{Cl})$ :

$$
G_{1}(y)=\frac{1}{2} y^{2}
$$

In this case, the contrast functions becomes essentially a measure of the fourth statistical moment of the source - kurtosis. As its value in case of the Gaussian variable equals zero, by maximizing the kurtosis of each of the sources, we ensure their independence.

- logarithm (C2):

$$
G_{2}(y)=\log (0.05+y)
$$

- square root $(C 3)$ :

$$
G_{3}(y)=\sqrt{0.05+y}
$$

Being a slowly growing nonlinear functions (Fig.2), $G_{2}(y)$ and $G_{3}(y)$ allow more robust estimation with respect to the outliers.

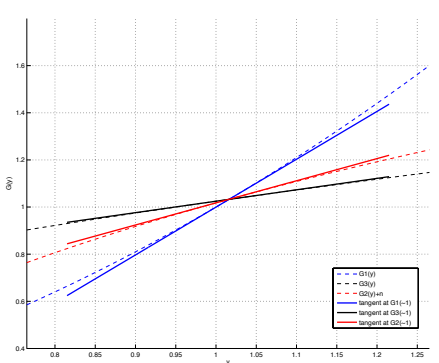

Fig. 2. Nonlinear functions

Additionally, by including the pseudo-covariance matrix of the observation target vectors in maximizing the contrast function (Eq. 2), the applied algorithm is generalized to the case of complex sources having a noncircular distribution [2]. This way, despite the modulus in equation 2 , the phase information is preserved.

The result of the incoherent target decomposition is the set of target vectors representing elementary scatterers and a set of scalars, providing their proportion in the total scattering. In our case, the target vectors of the independent scatterers are the columns of the estimated (de-whitened) mixing matrix $\mathbf{A}$. The contributions to the total backscattering are computed as the $\ell^{2}$ norms of the mixing matrix columns.

\subsection{TSVM Parametrization}

Being based on Kennaugh-Huynen condiagonalization projected onto the Pauli basis, the TSVM [3] allows parametrization of the target vector in terms of rotation angle $(\psi)$, maximum amplitude $(m)$, target helicity $\left(\tau_{m}\right)$, symmetric scattering type magnitude $\left(\alpha_{s}\right)$ and symmetric scattering type phase $\left(\Phi_{\alpha_{s}}\right)$, among which the last four are roll-invariant: 


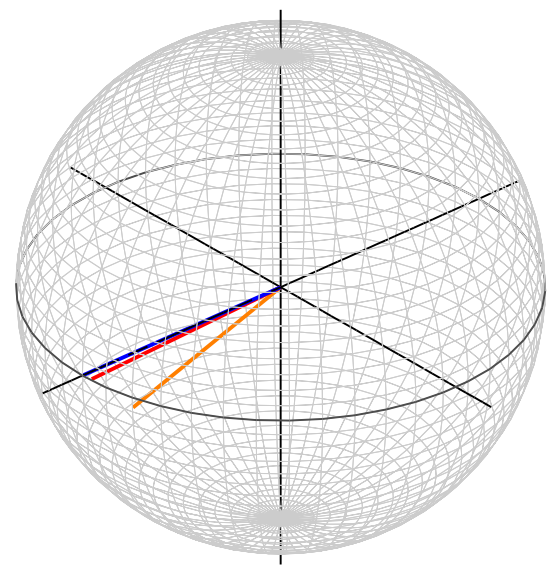

(a)

\begin{tabular}{|c|c|c|c|}
\hline Comp. & $\tau_{m}\left[^{\circ}\right]$ & $\alpha_{s}\left[^{\circ}\right]$ & $\left.\Phi_{\alpha_{s}}{ }^{\circ}\right]$ \\
\hline \hline 1st & $\mathbf{- 0 . 3 3 0 3}$ & $\mathbf{1 . 4 9 3 5}$ & $\mathbf{7 . 5 3 7 1}$ \\
\hline 2nd & -0.4230 & 7.3886 & -9.5901 \\
\hline 3rd & 8.0126 & 23.4325 & -83.7107 \\
\hline trihedral & 0 & 0 & {$[-90,+90]$} \\
\hline
\end{tabular}

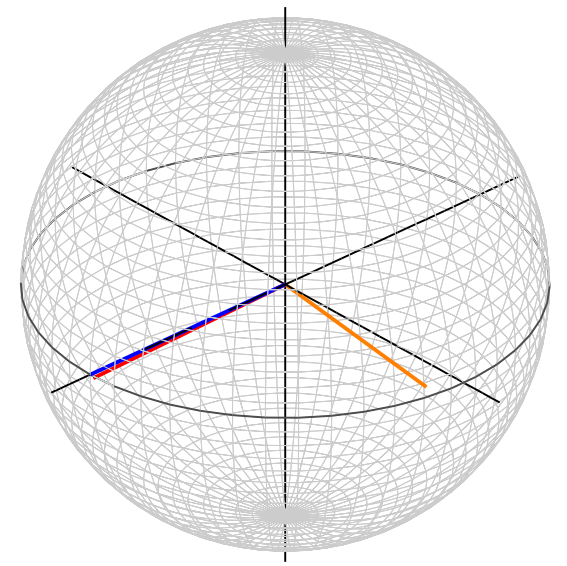

(b)

\begin{tabular}{|c|c|c|c|}
\hline Comp. & $\tau_{m}\left[{ }^{\circ}\right]$ & $\alpha_{s}\left[^{\circ}\right]$ & $\left.\Phi_{\alpha_{s}}{ }^{\circ}\right]$ \\
\hline \hline 1st & $\mathbf{- 0 . 2 8 0 1}$ & $\mathbf{0 . 5 3 2 4}$ & $\mathbf{- 2 7 . 4 1 5 2}$ \\
\hline 2nd & -0.2415 & 39.9067 & 2.5568 \\
\hline 3rd & 19.8393 & 58.4871 & 77.9176 \\
\hline trihedral & 0 & 0 & {$[-90,+90]$} \\
\hline
\end{tabular}

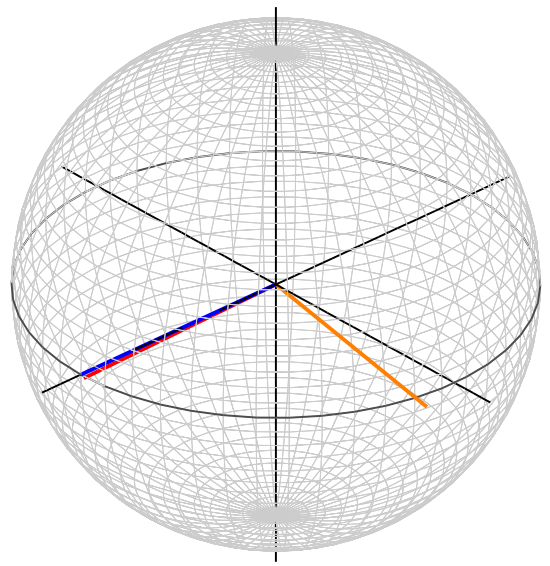

(c)

\begin{tabular}{|c|c|c|c|}
\hline Comp. & $\tau_{m}\left[{ }^{\circ}\right]$ & $\alpha_{s}\left[^{\circ}\right]$ & $\Phi_{\alpha_{s}}\left[^{\circ}\right]$ \\
\hline \hline 1st & $\mathbf{- 0 . 2 8 3 8}$ & $\mathbf{0 . 5 2 8 5}$ & $\mathbf{- 2 7 . 7 0 8 7}$ \\
\hline 2nd & -0.3573 & 41.1999 & -3.3304 \\
\hline 3rd & 5.7724 & 54.9698 & -68.5918 \\
\hline trihedral & 0 & 0 & {$[-90,+90]$} \\
\hline
\end{tabular}

Fig. 3. Symmetric target Poincaré sphere representation of the independent scattering components (1st - red, 2nd - orange) estimated in the class 8 with respect to trihedral (blue): (a) C1, (b) C2, (c) C3.

$$
\mathbf{k}=m|\mathbf{k}|_{m} e^{j \Phi_{s}}\left[\begin{array}{ccc}
1 & 0 & 0 \\
0 & \cos 2 \psi & -\sin 2 \psi \\
0 & \sin 2 \psi & \cos 2 \psi
\end{array}\right]\left[\begin{array}{c}
\cos \alpha_{s} \cos 2 \tau_{m} \\
\sin \alpha_{s} e^{j \Phi_{\alpha_{s}}} \\
-j \cos \alpha_{s} \sin 2 \tau_{m}
\end{array}\right]
$$

Using these parameters, it is eventually possible to represent the obtained independent target vectors on either symmetric or non-symmetric target Poincaré sphere. In our case, they do not necessarily form an orthogonal basis.

\section{RESULTS AND CONCLUSIONS}

The presented results (Fig. 3 and 4) are obtained by applying the ICA based ICTD to the RAMSES POLSAR X-band image acquired over Brétigny, France, illustrated with the Pauli colour coded image in Fig. 1. The same figure shows the classification map used to define sets of observation data for the ICA algorithm.

The first point of comparison between the proposed criteria in complex IC derivation $(C l, C 2$ and $C 3)$ is the possibility of identifying the class of trihedral reflectors present in the scene (Class 8 in Fig. 1.b). The mask derived from the classification map allowed us to select the set of the observation data containing target vectors from the regions in the image where the reflectors were placed. Further, one mixing matrix is estimated using each of three criteria. In each case, the first and the second dominant components are presented on symmetric scattering target Poincaré sphere [3] (Fig. 3). The third component parameters are provided in the tables but, due to the values of helicity and symmetric scattering type phase, it is not possible to illustrate it using a sphere.
The method is able to almost perfectly identify the class corresponding to the trihedral reflectors placed in the scene (Fig. 3). A curious fact is that the second dominant component in this case appears to be symmetric as well. Kurtosis criterion (Fig. 3.a) results in both components almost matching trihedral which indicates apparent "splitting" of the trihedral on two dominant components (Fig. 4). On the other side, in case of logarithm and square root criteria, the second component, although symmetric, rather represents weaker dipole backscattering. This is reflected through the increased value in entropy for $\mathrm{Cl}$ with respect to the other criteria.

The second point of comparison assumes the discrimination between different targets (a priori defined classes) in terms of the derived parameters and the entropy estimation. In Fig. 4 we illustrate the maps of the obtained roll-invariant parameters of the most dominant component for each of the three criteria, as well as the corresponding entropy images.

We can observe the better performances of kurtosis in discriminating between different targets, in the context of symmetry and symmetric scattering type magnitude. However, as already suggested, the entropy estimation scheme appears to be far better with the other two methods ( $C 2$ and $C 3$ ).

The overall performance of the analyzed ICA criteria in the frame of ICTD, seems to depend directly on the growth rate of the used nonlinear function. The ICA based on slowly growing nonlinear functions (logarithm and square root) (Fig. 2) are more efficient in both identifying trihedral as the most dominant backscattering mechanism and, although it is an implication, in estimating entropy. The drawback is not as good discrimination between the different classes as the one 


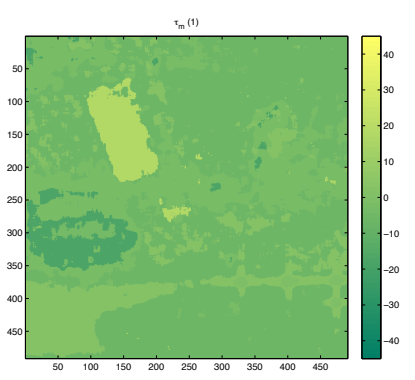

(a)

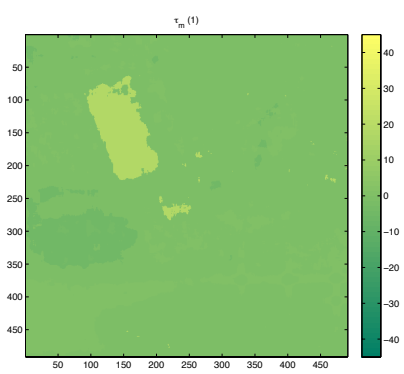

(e)

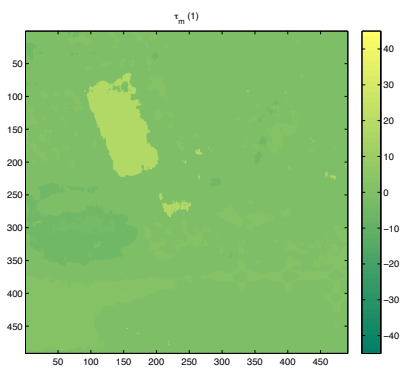

(i)

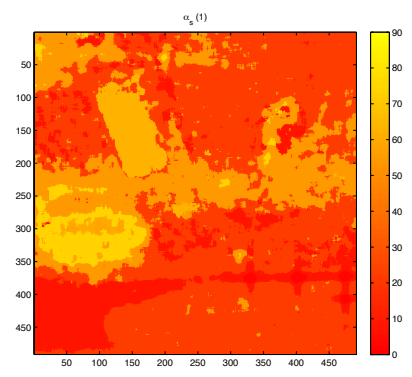

(b)

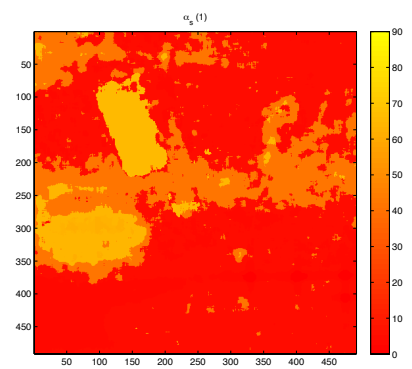

(f)

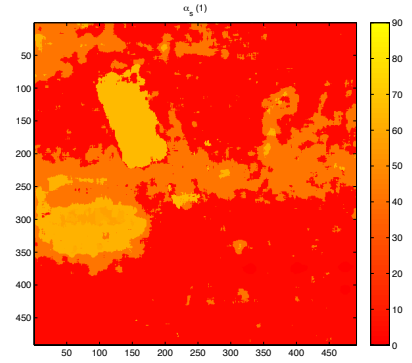

(j)

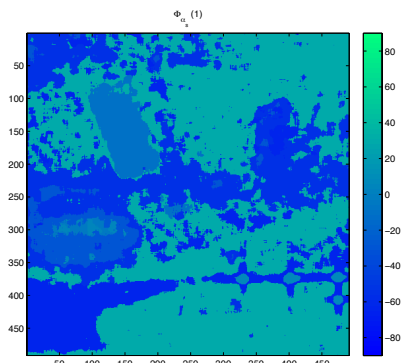

(c)

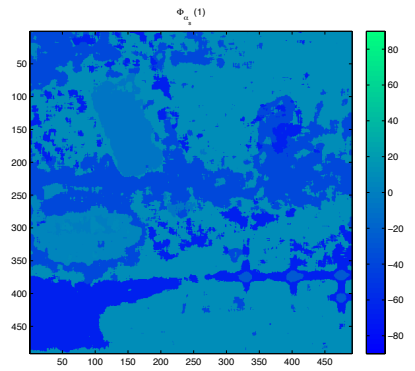

$(\mathrm{g})$

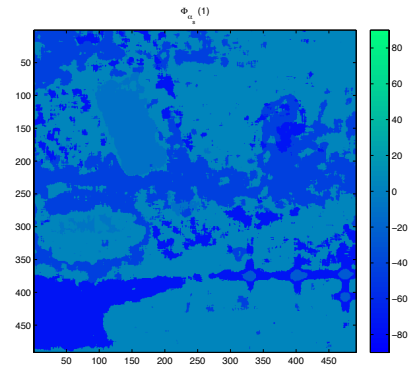

(k)

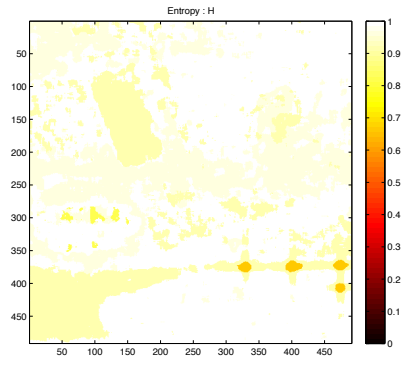

(d)

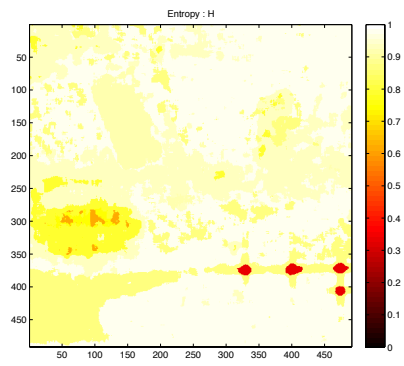

(h)

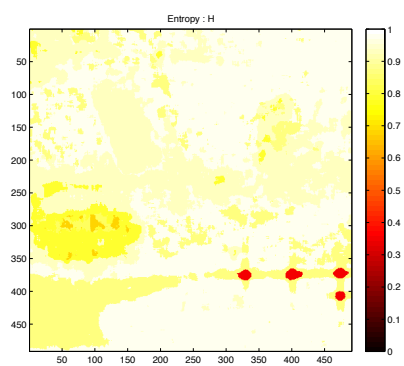

(1)

Fig. 4. The ICA based ICTD: $\tau_{m}(1)$ - helicity of the dominant comp. (a) C1 (e) C2 (i) C3; $\alpha_{s}(1)$ - symmetric scattering type magnitude of the dominant comp. (b) $\mathrm{C} 1$ (f) $\mathrm{C} 2$ (j) $\mathrm{C} 3 ; \Phi_{\alpha_{s}}(1)$ - symmetric scattering type phase of the dominant comp. (c) $\mathrm{C} 1$ (g) C2 (k) C3; H - entropy (d) C1 (h) C2 (l) C3.

achieved with the kurtosis criterion.

\section{REFERENCES}

[1] E. Bingham and A. Hyvrinen, "A fast fixed-point algorithm for independent component analysis of complex valued signals," Int. J. Neural Syst., vol. 10, no. 1, pp. 18, 2000.

[2] M. Novey and T. Adali, "On extending the complex fastica algorithm to noncircular sources," IEEE Trans. Signal Processing, vol. 56, no. 5, pp. 2148-2154, 2008.

[3] R. Touzi, "Target scattering decomposition in terms of roll-invariant target properties," IEEE Trans. Geosci. Remote Sens., vol. 45, no. 1, pp. 73-84, 2007.

[4] D. Massonet and J.-C. Souyris, Imaging with Synthetic Aperture Radar, CRC Press, Taylor and Francis Group, Boca Raton, FL, USA, 2008.

[5] S. R. Cloude and E. Pottier, "A review of target decomposition theorems in radar polarimetry," IEEE Trans. Geosci. Remote Sens., vol. 34, no. 2, pp. 498-518, 1996 ,
[6] A. Hyvarinen and E. Oja, "Independent component analysis: Algorithms and applications," Neural Networks, vol. 13, no. 4-5, pp. 411430, 2000.

[7] F. Totir, G. Vasile, L. Bombrun, and M. Gay, "Polsar images characterization through blind source separation techniques," in Proc. IGARSS, Hawaii, USA, 2010, pp. 4039-4042.

[8] N. Besic, G. Vasile, J. Chanussot, and S. Stankovic, "Poincare sphere representation of independent scattering sources: application on distributed targets," in ESA SP-713 - POLinSAR 2013, Frascati, IT, 2013.

[9] P. Formont, F. Pascal, G. Vasile, J.-P. Ovarlez, and L. Ferro-Famil, "Statistical classification for heterogeneous polarimetric sar images," IEEE J. Sel. Topics Signal Process., vol. 5, no. 3, pp. 398-407, 2011.

[10] G. Vasile, J. P. Ovarlez, F. Pascal, and C. Tison, "Coherency matrix estimation of heterogeneous clutter in high-resolution polarimetric sar images," IEEE Trans. Geosci. Remote Sens., vol. 48, no. 4, pp. 1809$1826,2010$.

[11] A. Hyvarinen, "Fast and robust fixed-point algorithms for independent component analysis," IEEE Trans. on Neural Netw., vol. 10, no. 3, pp. 626634, 1999. 